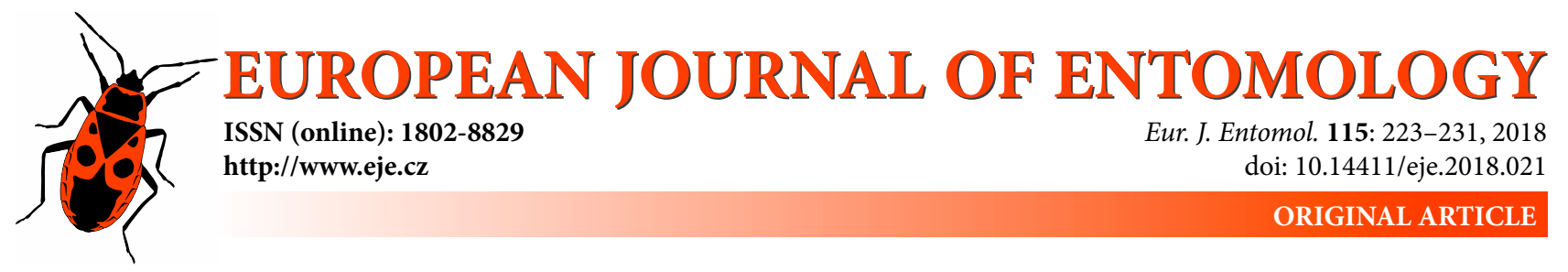

\title{
RNA interference mediated knockdown of an inhibitor of apoptosis protein induces apoptosis in Mythimna separata (Lepidoptera: Noctuidae)
}

\author{
MASASH KAMEZAKI*, KAKERU YOKOI** and Ken MIURA \\ Department of Applied Entomology, Graduate School of Bio-agricultural Sciences, Nagoya University, Chikusa, \\ Nagoya 464-8601, Japan; e-mails: kkame@agr.nagoya-u.ac.jp, yokoi123@affrc.go.jp, k-miura@agr.nagoya-u.ac.jp
}

Key words. Lepidoptera, Noctuidae, Mythimna separata, apoptosis, hemocytes, caspase, cDNA, RNA interference, inhibitor of apoptosis protein, TUNEL strain

\begin{abstract}
Coordinated regulation of apoptosis is critical for development, homeostasis, and immunity in larvae of Metazoa. We determined the full nucleotide sequence of an inhibitor of an apoptosis protein in a lepidopteran insect Mythimna separata (Walker) (MsIAP) and carried out functional analyses of the MsIAP. The full-length cDNA of MsIAP was 1642 bp, which encoded 379 amino acid residues with a calculated molecular mass of 41,834 Da, and two BIR domains and one RING domain revealed using amino acid sequence analysis. In addition, the sequences of these domains were similar to Drosophila IAP1 and those of some other lepidopteran insects. We carried out a functional analyses of MsIAP related to apoptosis regulation using RNA interference. The effects of MsIAP knockdown on adhering hemocytes and non-adhering hemocytes as controls were examined using Hoechst33342/propidium iodide staining, effector caspase activity and terminal deoxynucleotidyl transferase-mediated dUTP nick-end labelling (TUNEL) staining. A significantly higher number of propidium iodide and TUNEL-positive cells was recorded in adhering hemocytes from MsIAP knockdown larvae than from control larvae, but these differences were not recorded for non-adhering hemocytes. However, higher effector caspase activity was detected in both adhering and non-adhering hemocytes from MsIAP knockdown larvae compared to that in control larvae. These results indicate that the knockdown of MsIAP induces apoptosis in larval adhering hemocytes, which MsIAP negatively and non-redundantly regulate apoptosis, and that IAP function is conserved in $M$. separata and other insect species including Drosophila and several lepidopteran insects.
\end{abstract}

\section{INTRODUCTION}

Apoptosis is a genetically programmed cellular process that removes unwanted, damaged, or infected cells by inducing "suicide" in these cells (Clarke, 1990). Thus, apoptosis has a crucial role in development, homeostasis and immunity (Vaux \& Korsmeyer, 1999; Opferman \& Korsmeyer, 2003). The molecular mechanism of apoptosis was initially investigated in the model nematode Caenorhabditis elegans (Maupas), which has a protease ced-3 and nuclease nuc-1 that function as positive and negative regulators of apoptosis, respectively (Horvitz, 2003; Riedl \& Shi, 2004). Drosophila melanogaster (Meigen) and mammals have more complex cell death mechanisms. Multiple family ced-3 protease homologs, which are known as caspases, have central roles in regulating apoptosis. Caspases fall into two categories: initiator caspases are activated first and then cleave downstream effector caspases, which degrade several distinct substrates leading to cell death. During the cell death process, a series of morphologically recognizable changes, such as cell shrinkage, chromatin condensation, genomic DNA fragmentation, membrane blebbing and the emergence of apoptotic bodies, occur (Wyllie et al., 1980).

Inhibitor of apoptosis proteins (IAPs) are well-studied negative regulators of apoptosis. IAPs bind directly with both initiator and effector caspases and block their activities (Salvesen \& Duckett, 2002; Riedl \& Shi, 2004; Vaux $\&$ Silke, 2005). For example, Drosophila IAP1 (DIAP1), an IAP family protein in D. melanogaster, is a key negative regulator of apoptosis and inhibits DRONC (D. mela-

\footnotetext{
* Present address: Biology Group, Discovery Health and Crop Sciences Research Laboratory, Sumitomo Chemical Co., Ltd, 4-2-1, Takarazuka 665-8555, Japan

** Corresponding and present address: Insect Genome Research and Engineering Unit, Division of Applied Genetics, Institute of Agrobiological Sciences, National Agriculture and Food Research Organization (NARO), 1-2 Owashi, Tsukuba, Ibaraki, 305-8634, Japan; e-mail: yokoi123@affrc.go.jp
} 
nogaster initiator of caspase), drICE and DCP-1 (D. melanogaster effector caspases) (Kornbluth \& White, 2005; Tenev et al., 2005). Most IAP family proteins have two important domains, a baculoviral IAP repeat (BIR) domain and really interesting new gene (RING) domain (Riedl \& Shi, 2004; Vaux \& Silke, 2005). The BIR domain is a $\mathrm{Zn}^{2+}$ binding domain and consists of approximately 70 amino acid residues. This domain interacts with effector or initiator caspases. The RING domain is a $\mathrm{Zn}^{2+}$-binding domain with E3 ubiquitin ligase activity and is involved in the negative regulation of apoptosis.

In several species of Lepidoptera, IAP orthologues function as negative regulators of apoptosis, which suggests that roles of IAPs are conserved in mammals, D. melanogaster and lepidopteran insects (Huang et al., 2000, 2001; Liao et al., 2002). We investigated whether the function of an IAP orthologue in the lepidopteran insect, Mythimna separata (Walker) (MsIAP), is conserved. Previously, we examined its sensitivity to RNAi in multiple tissues of $M$. separata (Yokoi et al., 2013). Using a partial sequence of MsIAP, we found that MSIAP mRNA was ubiquitously expressed and that significant gene silencing of MSIAP was achieved only in adhering hemocytes from isolated abdomens treated with $M s I A P$ double-stranded RNA (dsRNA). Here, we determined the full cDNA sequence of MSIAP and the predicted MSIAP amino acid sequence was analyzed. MSIAP function was determined in terms of cell viability, effector caspase activity and genomic DNA fragmentation. We found that MSIAP functioned as a negative regulator of apoptosis in adhering hemocytes, which indicates that the function of IAP as a negative regulator of apoptosis is conserved in $M$. separata.

\section{MATERIALS AND METHODS}

\section{Insect rearing and preparation of isolated abdomens}

M. separata was reared as described in Suzuki \& Tanaka (2007) and the method of preparing isolated abdomens is described in Yokoi et al. (2013).

\section{First strand cDNA synthesis for RT-PCR}

Total hemocyte RNA was used for 1st strand cDNA synthesis using PrimeScript reverse transcriptase (TAKARA) and oligo(dT) 20 primer. The 1 st strand cDNA library was used as a template for RT-PCR with a pair of degenerate primers. A pair of degenerate primers were designed based on the highly conserved regions identified by alignment of several amino acid sequences of published insect IAPs using the Clustal W 1.83 program (Thompson et al., 1994). Sequences of the degenerate primer pair were: IAP-F1d, 5'-ATGAARACNTTYGARAARTGGC-3'; IAP-R1d, 5'-TCYTCNGCRWARCAIATYTTICA-3'. The locations of these degenerate primers in MSIAP are shown in Fig. 1. The RT-PCR reaction mixture was first denatured at $94^{\circ} \mathrm{C}$ for 2 min, followed by 35 cycles of denaturation at $94^{\circ} \mathrm{C}$ for $30 \mathrm{~s}$, annealing at $50^{\circ} \mathrm{C}$ for $1 \mathrm{~min}$ and elongation at $72^{\circ} \mathrm{C}$ for $30 \mathrm{~s}$. The PCR products were separated on a $1.8 \%$ agarose gel. The DNA bands of the expected sizes were excised and recovered using a QIAquick Gel Extraction Kit (QIAGEN). DNA fragments were checked for integrity and amount using gel electrophoresis, sub cloned into the pCR2.1 vector (Invitrogen), and sequenced with an ABI Prism Dye Terminator Cycle Sequencing Kit (Applied Biosystems) and a DNA sequencer (Model 3130, Applied Bio- systems). BLASTx search (URL: https://blast.ncbi.nlm.nih.gov/ Blast.cgi?PROGRAM=blastx\&PAGE_TYPE=BlastSearch) confirmed that the obtained DNA fragment encoded an amino acid sequence that is very similar to known insect IAPs.

\section{3' and 5' RACE}

For 3' RACE, the 1st strand cDNA synthesis was primed with a tagged oligo-(dT) primer (5'-CTACAGTCTGCTCACAGCATAGTATTTTTTTTTTTTTTTTTTTTTTTTTVN-3'). The 3' region of MsIAP was amplified with gene-specific primers (5'-ACGGAGGCTTGAAAGACTGGGAG-3') and a 3' anchor primer (5'-CTACAGTCTGCTCACAGCATAGTA-3') using thermal cycling conditions of initial denaturation at $94^{\circ} \mathrm{C}$ for $2 \mathrm{~min}$, followed by 35 cycles of denaturation at $94^{\circ} \mathrm{C}$ for $30 \mathrm{~s}$, annealing at $68^{\circ} \mathrm{C}$ for $30 \mathrm{~s}$ and elongation at $72^{\circ} \mathrm{C}$ for 1 min 30 s. For 5' RACE, a RNA oligo (5'-AAGCAGUGGUAUCAACGCAGAGUGGG-3') was included in the reverse transcription reaction and the 3'-tagged 1st strand cDNA pool was subjected to RT-PCR with a 5' anchor primer (AAGCAGTGGTATCAACGCAGAGT-3') and gene-specific primer (5'-GCGAGCTTGAACCTCGGCAAT-3'). The locations of the two gene-specific PCR primers used for the RACE procedures and degenerate primers are shown in Fig. 1. Thermal cycling conditions used were an initial denaturation step at $94^{\circ} \mathrm{C}$ for $2 \mathrm{~min}$, followed by 35 cycles of denaturation at $94^{\circ} \mathrm{C}$ for $30 \mathrm{~s}$, annealing at $68^{\circ} \mathrm{C}$ for $30 \mathrm{~s}$ and elongation at $72^{\circ} \mathrm{C}$ for $1 \mathrm{~min} 30 \mathrm{~s}$. The amplification products were fractionated by agarose gel electrophoresis and specifically-amplified DNA fragments were recovered and sequenced directly. In some cases, the DNA fragments were sequenced directly with the same reagents and device described previously and the sequences assembled into the full MsIAP nucleotide sequence. The full MSIAP nucleotide sequence was deposited in GenBank (accession no. AB778567).

\section{RNA interference}

The materials and methods related to RNAi were the same as in Yokoi et al. (2013). Briefly, the DNA templates for dsRNA synthesis were prepared using conventional PCR with the pairs of T7-tagged PCR primers for the respective factors, for which the locations in MsIAP are shown in Fig. 1. The sequences of the primers were: 5'-taatacgactcactatagggagaACTTTCCTTTCTGGAGATGAACT-3' (sense) and 5'-taatacgactcactatagggagaTCATCACCTTCTGGACGTACTC-3' (anti-sense). Synthesis of dsRNA was carried out using a MEGAscript RNAi kit (Ambion) with these templates. The dsRNA fragment was purified according to the manufacturer's instructions, and the integrity and amount were assessed using agarose gel electrophoresis and spectrophotometry, respectively. For the production of dsRNA with unrelated sequences, enhanced green fluorescent protein (EGFP) sequence-derived dsRNA was prepared in the same manner using the pEGFP-N1 plasmid (Clontech) as a PCR template.

For IAP gene function analysis, we prepared isolated abdomens using day 16 th instar larvae. The detailed procedures for preparing and isolating abdomens and dsRNA injection are described by Yokoi et al. (2013). Briefly, the larvae were constricted between the third thoracic segment and the first abdominal segment with a nylon thread, and the posterior part minus two pairs of haematopoietic organs was used as an isolated abdomen. dsRNA was injected into isolated abdomens $24 \mathrm{~h}$ post ligation. We performed a preliminary assay for IAP knockdown effects on isolated abdomens. We found that when $3 \mu \mathrm{g}$ dsRNA MsIAP was injected into an isolated abdomen and the isolated abdomen was incubated for $48 \mathrm{~h}$, there was approximately a $60 \%$ reduction in MSIAP mRNA in adhering hemocytes compared to that in control hemocytes, but no significant effects of MsIAP knockdown was recorded in 


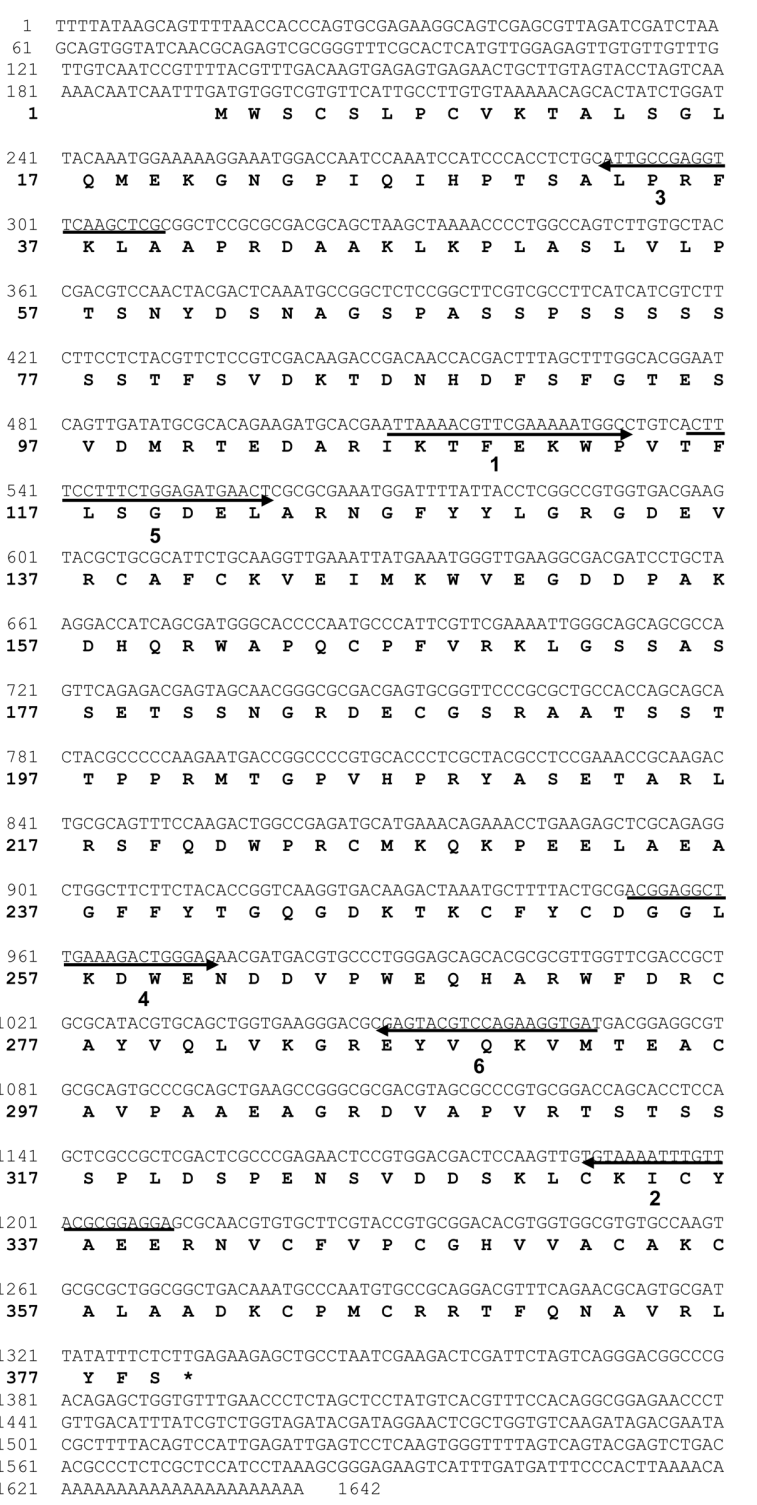

Fig. 1. CDNA and predicted amino acid sequences of MsIAP. The full-length cDNA sequence was assembled from partial sequences obtained using RT-PCR with degenerate primers, 3' RACE and 5 RACE. Arrows with numerals 1 to 6 indicate the positions of primers used: 1 and 2, a pair of degenerate primers for RT-PCR; 3 , gene-specific antisense primer for 5' RACE; 4, gene-specific sense primer for 3' RACE; 5 and 6, T7-tagged primer pair for in vitro transcription template preparation. An asterisk denotes the stop codon. Nucleotide and amino acid residue numbers are shown on the left and right sides of each line.

non-adhering hemocytes, which is similar to the results of Yokoi et al. (2013). Therefore, we used these conditions for MsIAP knockdown of hemocytes.

\section{Hoechst33342 and propidium iodide staining}

Hemocyte viability was assessed by plasma membrane integrity using double staining with Hoechst33342 and propidium iodide (PI). Hemolymph was diluted with 9 volumes of SF900 III medium (Thermo Fisher SCIENTIFIC) with $8 \%(\mathrm{v} / \mathrm{v})$ saturated phenyltiourea (PTU), which inhibits spontaneous melanization of the hemolymph and incubated on a glass slide (Matsunami, S2441) for $15 \mathrm{~min}$ at room temperature. The glass slide was washed three times with the same medium and non-adhering hemocytes, consisting of spherulocytes, prohemocytes and oenocytoids (Lavine \& Strand, 2002) were collected into a plastic tube and centrifuged at $600 \mathrm{~g}$ at $4^{\circ} \mathrm{C}$ for $10 \mathrm{~min}$. Adhering hemocytes, which remained on the glass slide, mainly consisted of granulocytes and plasmatocytes (Lavine \& Strand, 2002). The nonadhering hemocyte precipitate was resuspended in the same medium and the hemocyte suspension was put on a Mas-coated glass slide (Matsunami, S9441), which allows M. separata non-adhering hemocytes to adhere to the coated surface. Both adhering and non-adhering hemocytes on the glass surface were washed three times with PBS and double-stained with Hoechst33342 (10 mg/ $\mathrm{mL}$ in PBS) and PI ( $2 \mathrm{mg} / \mathrm{mL}$ in PBS) for $3 \mathrm{~min}$. The samples were washed briefly with PBS, immediately observed under a fluorescent microscope (Olympus, Model BX41) and imaged. The numbers of Hoechst-positive (all) cells and PI-positive (dead or late apoptotic) cells were counted at $200 \times$ magnification in at least six randomly selected fields.

\section{Caspase-3/7 activity assay}

The activity of effector caspase-3/7 was determined in larval hemocyte preparations using a Caspase-Glo 3/7 kit (Promega). The hemolymph from larvae pretreated with MSIAP or EGFP dsRNA was mixed with an equal volume of SF900 III with $8 \%$ (v/v) PTU and aliquoted into a 96-well plate. Fifteen minutes later, non-adhering hemocytes were transferred to another well. Hemocyte preparations were lysed in the presence of substrate and caspase activity determined using a luminometer (Turner Designs, Model TD 20/20). The caspase activities in the IAP knockdown and control of adhering and non-adhering hemocytes were determined. First, data normality for each category was tested using the Kolmogorov-Smirnov test. If significant normality of the two samples was detected, a Student's t test was used. If significant normality of the two samples was not detected, a MannWhitney U test was used.

\section{Terminal deoxynucleotidyl transferase-mediated dUTP nick-end labelling (TUNEL) assay}

Adhering and non-adhering hemocyte samples on glass slides were prepared as above. Hemocytes were labelled according to the protocol of an In Situ Cell Death Detection Kit, AP (Roche), samples were observed under a fluorescent microscope and imaged. The total numbers of cells and TUNEL-positive cells in both hemocyte samples were counted.

\section{Sequence analyses}

We used GENETYX ver. 9 software (GENETYX corporation) and InterPro (http://www.ebi.ac.uk/interpro/) (Hunter et al., 2009) to edit and analyze nucleotide and amino acid sequences. Homology searches were performed using BLAST at DNA Data Bank of Japan (http://blast.ddbj.nig.ac.jp).

\section{RESULTS}

\section{cDNA sequence determination and analyses of MsIAP}

The full cDNA sequence of MSIAP was obtained by a combination of PCR-based approaches. The MsIAP cDNA has 1642 nucleotides and encodes a polypeptide of 379 amino acid residues with a calculated molecular mass of 41,834 Da (Fig. 1).

The predicted amino acid sequence of MsIAP was aligned with the insect IAP amino acid sequences for Spodoptera frugiperda (Smith) IAP (SfIAP, accession no. AAF35285) (Huang et al., 2000), Tricoplusia ni (Hübner) IAP (TnIAP, accession no. AF195528) (Liao et al., 2002), Bombyx mori (Linnaeus) IAP (BmIAP, accession no. NP001037024) (Huang et al., 2001), and DIAP1 (accession no. Q24306), 
A
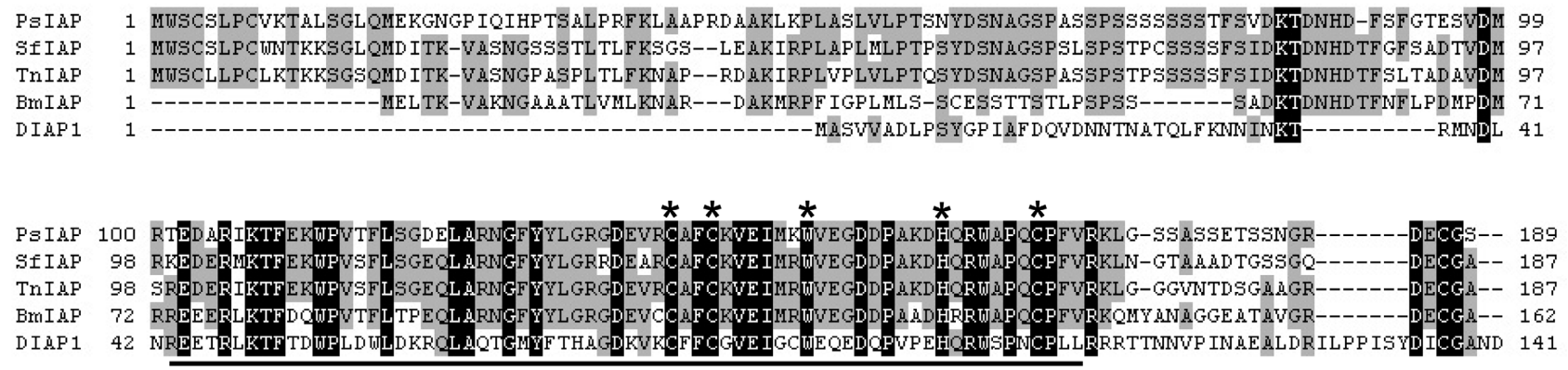

BIR1
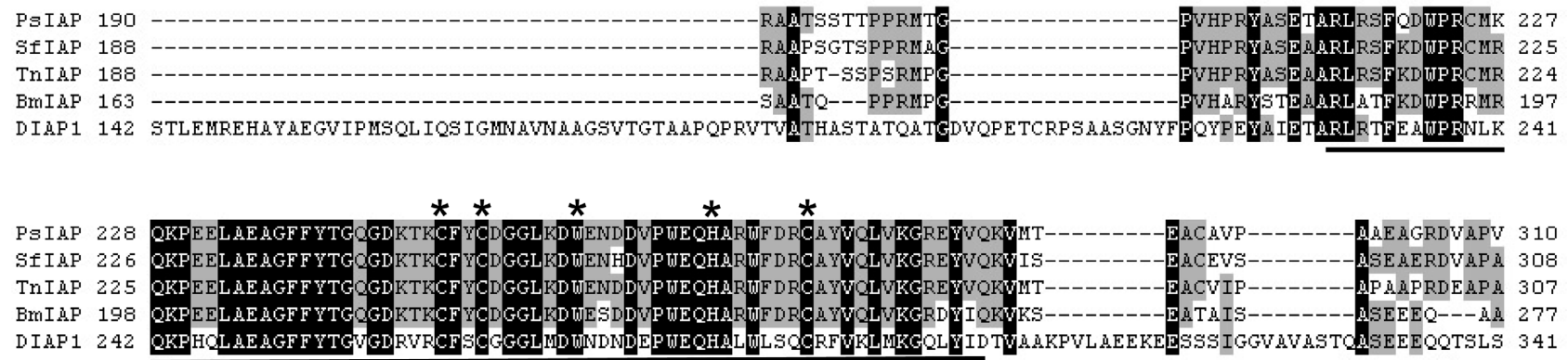

BIR2

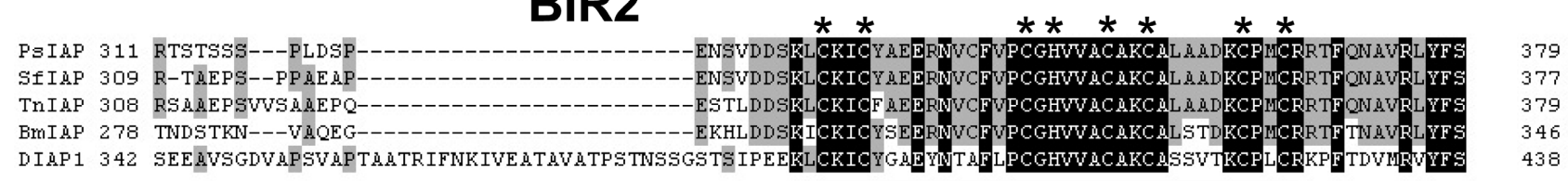

\section{RING}

B

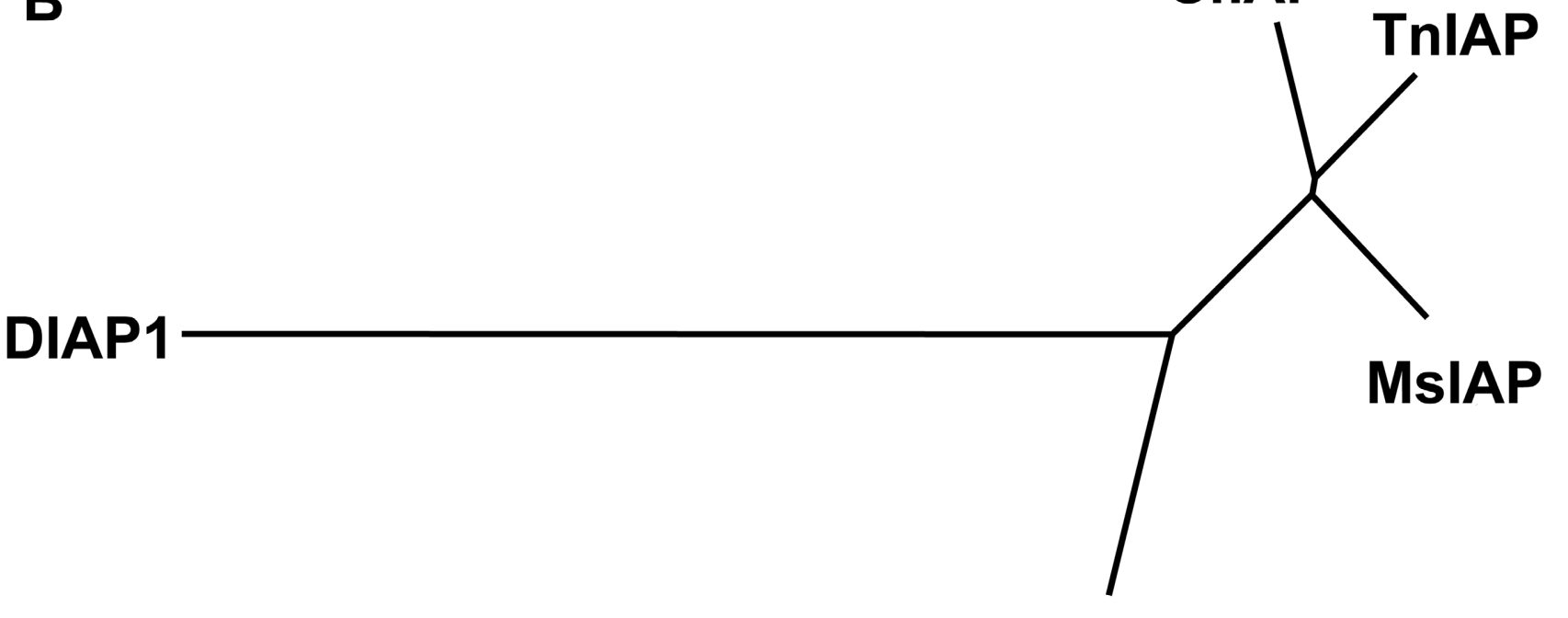

BmIAP

Fig. 2. Amino acid sequence alignment (A) and phylogenetic tree (B) of MsIAP and known insect IAPs. The Spodoptera frugiperda IAP (SfIAP, accession no. AAF35285), Tricoplusia ni IAP (TnIAP, accession no. AF195528), Bombyx mori IAP (BmIAP, accession no. NP001037024) and Drosophila melanogaster DIAP1 (accession no. Q24306) amino acid sequences were aligned together with MsIAP sequence using the Clustal W algorithm. In (A), residue numbers are shown on both sides of each line. Gaps introduced are indicated by hyphens. Two BIR domains and one RING domain are marked by thick horizontal bars. The amino acid residues conserved in all (five) sequences are shown in white letters on a black background while those found in three or four sequences are on a grey background. Asterisks indicate important residues in each domain. In (B), is the phylogenetic tree constructed based on sequence alignment data. The length of each branch represents the degree of difference between each sequence. 
A
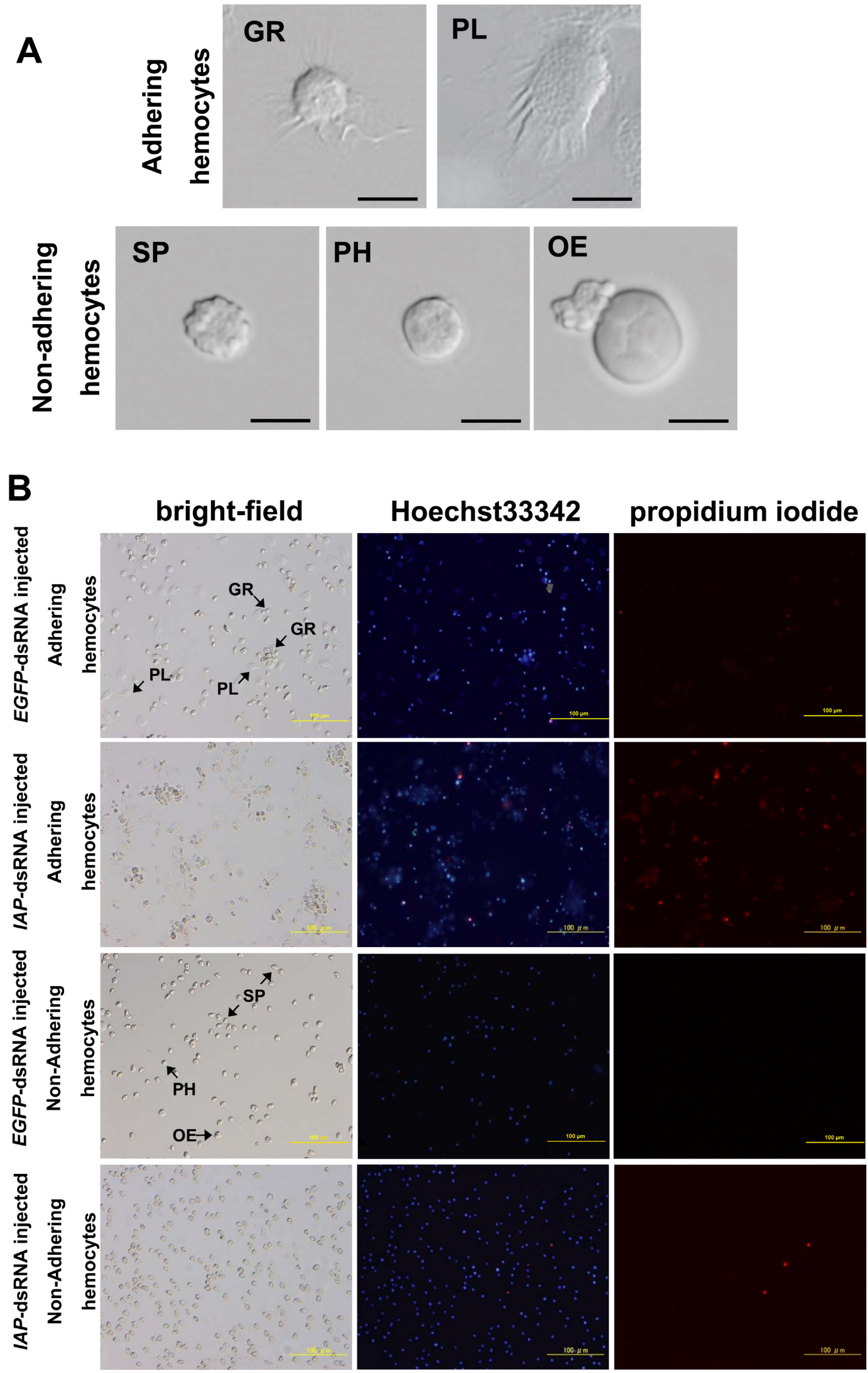

Fig. 3. Hemocyte viability after dsRNA treatment. A - Images of $M$. separata hemocyte species show adhering hemocytes, such as granulocytes (GR) and plasmatocytes (PL), and non-adhering hemocytes, such as spherulocytes (SP), prohemocytes (PH) and oenocytoids $(\mathrm{OE})$, under high magnification with $10-\mu \mathrm{m}$ scale bars. An adjacent SP is also present in the OE panel. B - Isolated larval abdomens were injected with dsRNA of either EGFP (top and third from top panels) or MsIAP (second from top and bottom panels). Forty-eight hours later, adhering and non-adhering hemocyte preparations were double-stained with Hoechst33342 and PI. Adhering hemocyte species with typical shapes are marked in the two upper left panels. Horizontal bars indicate a length of $100 \mu \mathrm{m}$. Note that dense PI staining of adhering hemocytes from MsIAP dsRNA-injected larvae was recorded. 


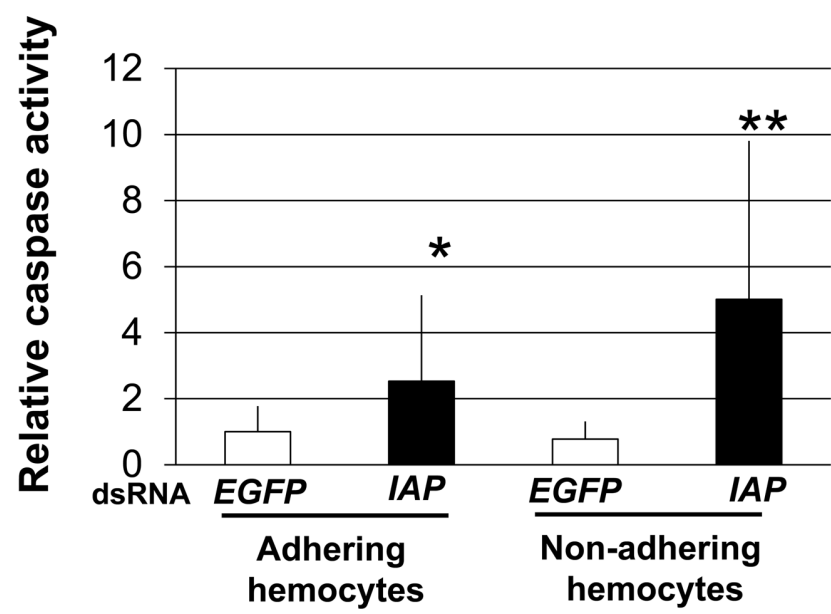

Fig. 4. Caspase activity in hemocytes treated with MsIAP or EGFP dsRNA. Isolated larval abdomens were treated as in Fig. 3, and caspase- $3 / 7$ activity was determined in adhering and non-adhering hemocyte preparations $48 \mathrm{~h}$ post-dsRNA injection. There were $6-8$ biological replicates in each sample. Each value is calculated relative to the average value of adhering hemocytes from EGFP dsRNA-injected larvae, which was set to 1 . Each vertical bar is a mean \pm S.D. Asterisk and double asterisks indicate $P=0.09$ using Student's t-test and $P=0.03$ using Mann-Whitney $U$ test, respectively.

which is the most similar to Lepidopteran IAPs (Hay et al., 1995) (Fig. 2A), and the phylogenetic tree was constructed using sequence alignment (Fig. 2B). The InterPro search of the predicted MsIAP revealed two BIR (BIR1 and BIR2 in Fig. 2A) domains and one RING domain (Hunter et al., 2009), and the sequences of these domains of MsIAP are similar to that in other insect IAPs. Lepidopteran IAPs formed a cluster in the phylogenetic tree except for DIAP1, which indicates that lepidopteran IAP sequences are conserved (Fig. 2B).

\section{Effects of MsIAP dsRNA injection on hemocyte viability}

Hemocytes were double-stained with Hoechst33342 and PI and observed using fluorescence microscopy. We counted the number of hemocytes double-stained with Hoechst33342 and PI or stained only with Hoechst33342 (Table 1). Typical images of the five hemocyte species are shown in Fig. 3A. Hemocyte spreading was observed in adhering hemocytes, such as granulocytes and plasmatocytes, but not in non-adhering hemocytes such as spherulocytes, prohemocytes and oenocytoids. Typical images of adhering and non-adhering hemocytes from isolated abdomens treated with EGFP or MSIAP dsRNA are shown in Fig. 3B. Adhering hemocytes consisted mainly of granulocytes and plasmatocytes, and non-adhering hemocytes consisted mainly of spherulocytes, prohemocytes and oenocytoids as indicated in the bright field images in Fig. 3B. For adhering hemocytes from EGFP dsRNA injected larvae, only a few hemocytes were PI-positive (dead or late apoptotic) (Fig. 3B, top panels and Table 1). MsIAP knockdown increased the number of PI-stained adhering hemocytes but not the number of non-adhering hemocytes (Fig. 3B, the second panels from top and Table 1). The numbers of Hoechst33342-positive and PI-positive hemo-
Table 1. Numbers of Hoechst33342-positive and propidium iodide positive hemocytes in dsIAP-injected and dsEGFP-injected larvae.

\begin{tabular}{|c|c|c|c|}
\hline $\begin{array}{l}\text { Sample larvae } \\
\text { replicate }\end{array}$ & $\begin{array}{c}\text { No. of } \\
\text { Hoechst33342- } \\
\text { positive hemocytes }\end{array}$ & $\begin{array}{l}\text { No. of propidium } \\
\text { iodide-positive } \\
\text { hemocytes }\end{array}$ & Biological \\
\hline \multicolumn{4}{|c|}{ (A) Adhering hemocytes } \\
\hline $\begin{array}{l}\text { dsEGFP-injected } \\
\text { dsIAP-injected }\end{array}$ & $\begin{array}{l}1834 \\
3632\end{array}$ & $\begin{array}{c}188 \\
1586\end{array}$ & $\begin{array}{c}8 \\
12\end{array}$ \\
\hline \multicolumn{4}{|c|}{$\mathrm{P}<2.2 \mathrm{e}-16$ by Fisher's Exact Test. } \\
\hline \multicolumn{4}{|c|}{ (B) Non-adhering hemocytes } \\
\hline $\begin{array}{l}\text { dsEGFP-injected } \\
\text { dsIAP-injected }\end{array}$ & $\begin{array}{l}455 \\
800\end{array}$ & $\begin{array}{l}40 \\
41\end{array}$ & $\begin{array}{l}6 \\
7\end{array}$ \\
\hline
\end{tabular}

$P=0.9956$ by Fisher's Exact Test.

cytes in each sample are shown in Table 1. Thus, we confirmed that injection of MsIAP dsRNA into isolated larval abdomens caused cell death of adhering hemocyte species. For non-adhering hemocytes there was no significant difference between the MSIAP dsRNA treatment and control samples (Fig. 3B, the third from top and bottom panels, and Table 1).

\section{dsRNA treatment and caspase activity}

MSIAP dsRNA treatment disrupted plasma membrane integrity and caused cell death in adhering hemocytes (Fig. 3 ), which indicates that knockdown of MSIAP induced apoptosis in these cells. We determined the activity of two effector caspases, caspase- 3 and caspase-7, in the apoptosis of hemocytes. Adhering and non-adhering hemocyte fractions were prepared from isolated larval abdomens treated with either EGFP or MSIAP dsRNA. Caspase activity in adhering hemocytes from MsIAP dsRNA-treated larvae was approximately 2-fold higher. A test of normality for the two samples was performed, and the $P$ values of MsIAP dsRNA-treated and control samples were 0.9977 and 0.8562 , respectively. Therefore, we tested the difference in caspase activities using a Student's $t$-test. At the 10\% significance level, a significant difference in caspase activities was recorded, which is consistent with the results of the Hoechst/ PI staining (Fig. 4). Surprisingly, MsIAP dsRNA treatment increased caspase activity in non-adhering hemocytes even though these hemocytes were PI-negative and a significant RNAi knockdown was not achieved (Fig. 4). The test of normality of the results for the MsIAP and dsRNA-treated and control samples had $P$ values of 0.6296 and 0.9804 , respectively. Therefore, a Mann-Whitney U test was used because significant normality in the MsIAP dsRNA-treated sample was not detected. A significant difference in caspase activities between the two categories was detected at the $10 \%$ significance level.

\section{TUNEL staining}

To further characterize the type of cell death caused by $M S I A P$ knockdown, hemocytes treated with dsRNAs were subjected to TUNEL staining, which detects genomic DNA fragmentation. Typical images of TUNEL staining for each sample are shown in Fig. 5. The results were similar to those obtained using PI staining (Fig. 3). Only adhering hemocyte samples prepared from MSIAP dsRNA-treated 
bright-field

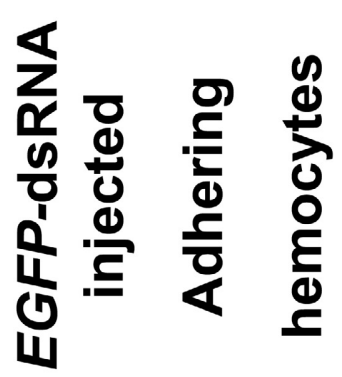

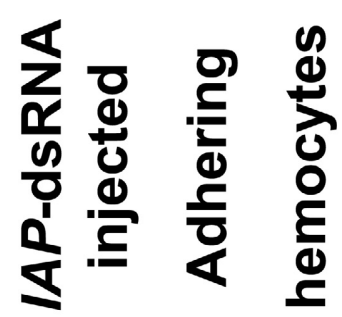
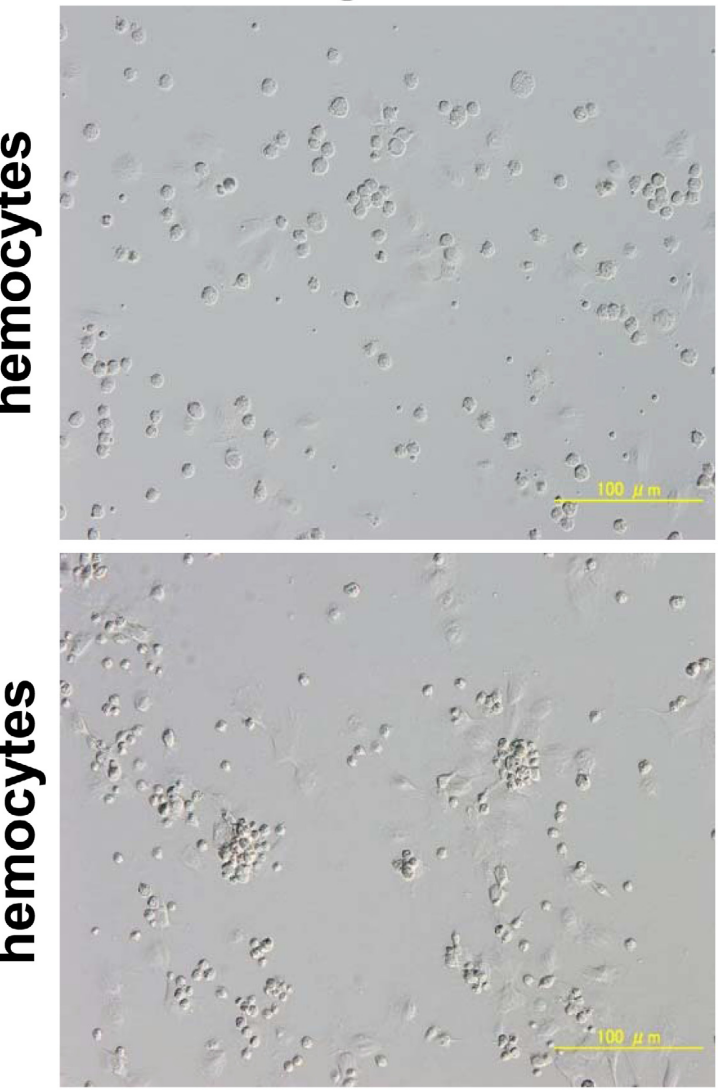

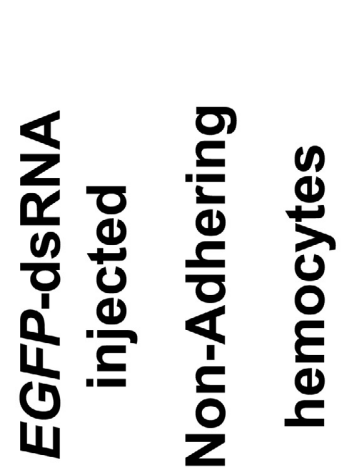

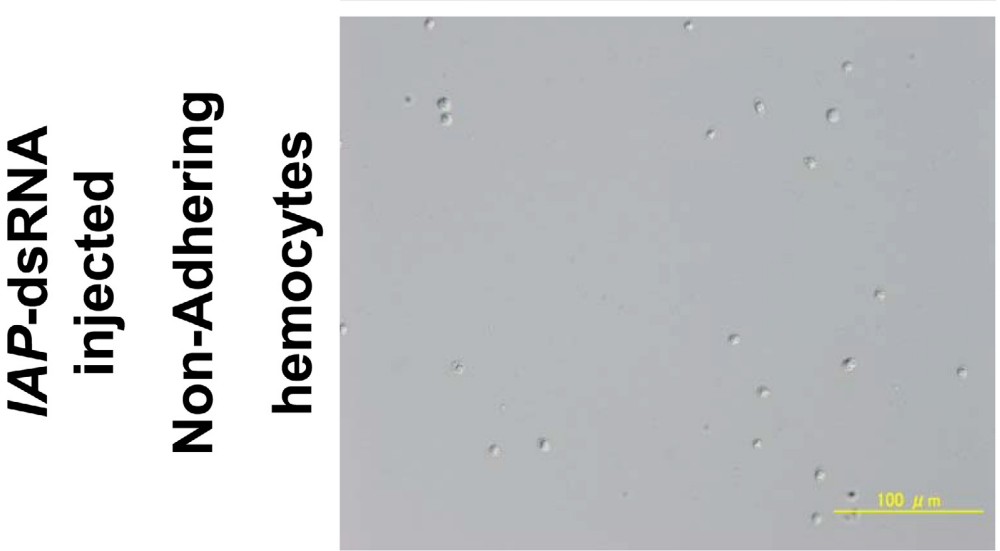

\section{TUNEL}
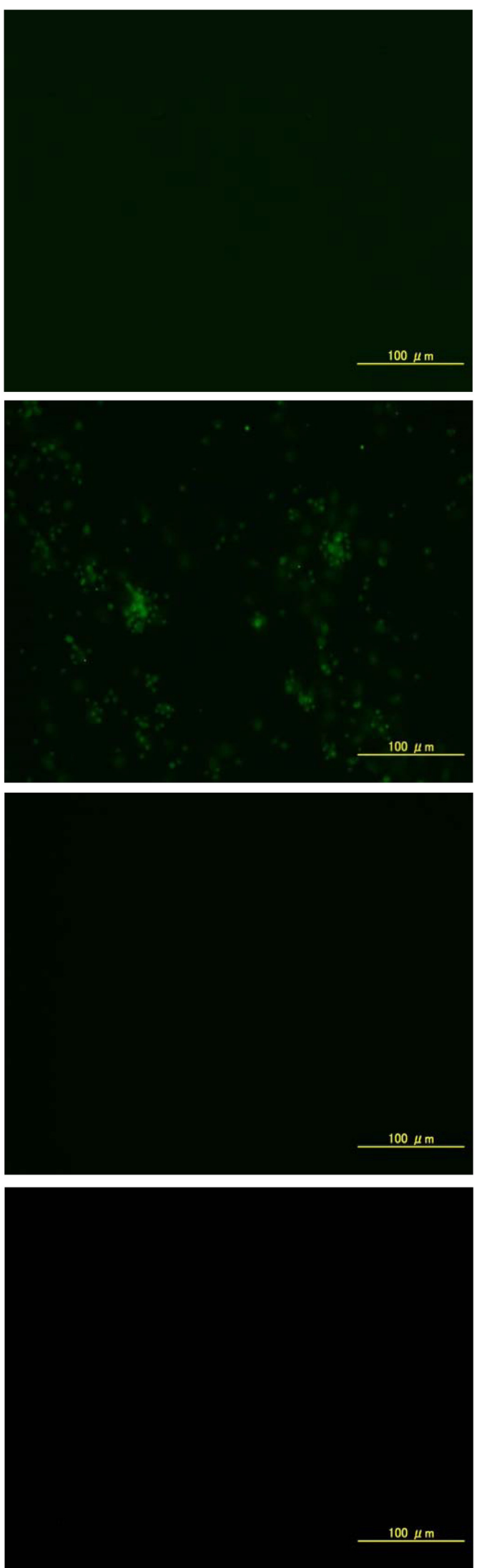

Fig. 5. TUNEL staining of hemocytes treated with MSIAP or EGFP dsRNA. Isolated abdomens of larvae were injected with either EGFP (top and third from top panels) or MsIAP dsRNA (second from top and bottom panels). Adhering and non-adhering hemocytes were prepared $48 \mathrm{~h}$ later and stained for fragmented genomic DNA using TUNEL. 
larvae had a high proportion of TUNEL-positive cells and other samples were TUNEL-negative. In addition, we counted the total and TUNEL-positive hemocyte numbers and present the ratio of TUNEL-positive hemocytes in Fig. 5. The ratio of the number of hemocyte cells to the number of TUNEL-positive cells in adhering hemocytes from dsEGFP-injected larvae and adhering hemocytes from dsMsIAP-injected larvae were 310/0 and 301/256, respectively. The ratio of the number of hemocyte cells to the number of TUNEL-positive cells in non-adhering hemocytes from dsEGFP-injected larvae and non-adhering hemocytes from dsMsIAP-injected larvae were $22 / 0$ and 26/0, respectively. Thus, TUNEL-positive cells were recorded only in adhering hemocytes treated with MsIAP dsRNA.

\section{DISCUSSION}

We determined the full nucleotide sequence of MSIAP and whether it functioned as a negative regulator of apoptosis using RNAi. Sequence analysis revealed that MsIAP had two BIR domains and one RING domain. A phylogenetic tree indicated that the amino acid sequence of MsIAP is similar to that of other lepidopteran IAP amino acid sequences. The zinc-binding BIR domain serves as an interface for protein-protein interactions, especially with caspases (Salvesen \& Duckett, 2002). The BIR1 and BIR2 domains of MsIAP both had the canonical pattern of three cysteines and one histidine, $\mathrm{CX}_{2} \mathrm{CX}_{6} \mathrm{WX}_{9} \mathrm{HX}_{6} \mathrm{C}$ (Deveraux \& Reed, 1999), similar to that in other insect IAPs. The RING domain is also found in IAPs, and IAPs with RING domains have ubiquitin protein ligase (E3) activity. The MsIAP RING domain has a C3HC4 pattern, which is conserved in other insect IAPs. Binding caspases undergo ubiquitination by the C-terminal RING domain, which has ubiquitin ligase activity (Meier et al., 2000; Riedl \& Shi, 2004; Vaux \& Silke, 2005). In lepidopteran insects, a few IAPs have been investigated, and all of them have two BIR domains and one RING domain, except for Bombyx IAP2 that has three BIR domains (Seshagiri et al., 1999; Huang et al., 2000, 2001; Vilaplana et al., 2007; Zhang et al., 2010). $S$. frugiperda and B. mori IAPs inhibit mammalian initiator caspase-9 but not effector caspase-3 or caspase-7 (Huang et al., 2000, 2001). In Drosophila, DIAP1 is essential for cell survival because the loss of DIAP1 leads to spontaneous initiation of apoptosis (Wang et al., 1999). DIAP1 also has two BIR domains, which are functionally distinct from each other. BIR1 mediates direct physical interaction with effector caspases, drICE and DCP-1, and BIR2 provides an interface for binding to the initiator caspase DRONC. Our sequence alignment and phylogenetic tree results reveal that MsIAP is similar to other lepidopteran IAPs, which indicates that MsIAP has the same target specificity as the IAPs of $S$. frugiperda and B. mori.

Because of the reduced mRNA levels of MSIAP in adhering hemocytes from isolated abdomens treated with MSIAP dsRNA (Yokoi et al., 2013), we determined viability using Hoechst/PI staining. Dead cells or cells with damaged plasma membranes were frequent among the ad- hering hemocytes from MsIAP dsRNA-injected isolated larval abdomens, and less so in samples from controls, which indicates that knockdown of MSIAP induces cell death in adhering hemocytes. We determined the activity of caspase-3 and caspase-7 using an apoptosis-specific TUNEL assay. Contrary to our expectations, the activities of effector caspase-3 and caspase-7 were higher in both adhering and non-adhering hemocytes from MsIAP dsRNAtreated larvae compared to those from controls. Apoptotic pathways in mammals and Drosophila are well conserved (Riedl \& Shi, 2004) so it is likely that the apoptotic pathways in $M$. separate are also conserved. Initiator caspase activation leads to effector caspase activation in mammals and Drosophila. Thus, the higher activities of caspase-3 and caspase-7 in adhering hemocytes from larvae treated with $M S I A P$ dsRNA could be due to activation of the $M$. separata caspase-9 homologue, which is a potential target of MSIAP. The percentage of TUNEL-stained adhering hemocytes recorded for MSIAP dsRNA-injected larvae was comparable to that of PI-positive cells, which clearly indicates that knockdown of MSIAP in hemocytes induced apoptosis. These results indicate that MsIAP negatively regulates apoptosis and that the function of IAP is conserved in mice, Drosophila and lepidopteran insects.

In mice, gene disruption of XIAP results in no major cell death phenotypes because its effect is compensated for by the upregulation of the related genes, $C I A P 1$ and $C I A P 2$ (Harlin et al., 2001). In mutant DIAP-deficient flies, cell death and death of embryos are reported (Martin, 2002), which indicates that DIAP is indispensable for cell survival. In the case of $M$. separata, knockdown of MsIAP induced apoptosis in adhering hemocytes, which indicates that MsIAP is a non-redundant factor that prevents unwanted apoptosis in this species.

Although injection of MSIAP dsRNA into isolated abdomens did not alter the amount of MSIAP mRNA in nonadhering hemocytes (Yokoi et al., 2013), a significantly higher activity of caspase- 3 and caspase- 7 was recorded. Higher activation of caspases may be due to an immune reaction. Caspases are involved in innate immune reactions in mammals (Meunier et al., 2014). Fig. 5 shows that numerous apoptotic adhering hemocytes from MsIAP knockdown occurred in hemolymph from isolated abdomens treated with MSIAP dsRNA. The immune reaction in non-adhering hemocytes may be induced by these apoptotic hemocytes, which activates caspases in the immune reaction by an unidentified mechanism.

In conclusion, we found an IAP orthologue in $M$. separata and the amino acid sequence of MsIAP is similar to that of other lepidopteran IAPs. In addition, we found that MsIAP functioned as a negative and non-redundant regulator of apoptosis, which indicates that the functions of IAP are conserved between Drosophila and lepidopteran insects including M. separata.

ACKNOWLEDGEMENTS. We thank T. Tanaka for his helpful technical advice. Also, we appreciate the advice of D. Kageyama, which greatly improved our manuscript. This work was 
supported in part by JSPS KAKENHI Grant Numbers 23658047 and 25450486 to $\mathrm{KM}$.

\section{REFERENCES}

Clarke P.G. 1990: Developmental cell death: morphological diversity and multiple mechanisms. - Anat. Embryol. (Berl). 181: 195-213.

DeverauX Q.L. \& ReED T.C. 1999: IAP family proteins - suppressors of apoptosis. - Genes Dev. 13: 239-252.

Eckelman B.P., Salvesen G.S. \& Scott F.L. 2006: Human inhibitor of apoptosis proteins: why XIAP is the black sheep of the family. - EMBO Rep. 7: 988-994.

Harlin H., RefFey S.B., Duckett C.S., Lindsten T. \& Thompson C.B. 2001: Characterization of XIAP-deficient mice. - Mol. Cell. Biol. 21: 3604-3608.

Hay B.A., Wassarman D.A. \& Rubin G.M. 1995: Drosophila homologs of baculovirus inhibitor of apoptosis proteins function to block cell death. - Cell 83: 1253-1262.

Horvitz H.R. 2003: Worms, life, and death (Nobel lecture). ChemBioChem 4: 697-711.

Huang Q.H., Deveraux Q.L., Maeda S., Salvesen G.S., StenNICKE H.R., Hammock B.D. \& Reed J.C. 2000: Evolutionary conservation of apoptosis mechanisms: Lepidopteran and baculoviral inhibitor of apoptosis proteins are inhibitors of mammalian caspase-9. - Proc. Natn. Acad. Sci. USA 97: 1427-1432.

Huang Q.H., Deveraux Q.L., Maeda S., Stennicke H.R., HamMOCK B.D. \& REED J.C. 2001: Cloning and characterization of an inhibitor of apoptosis protein (IAP) from Bombyx mori. Biochim. Biophys. Acta 1499: 191-198.

Hunter S., Apweiler R., Attwood T.K., Bairoch A., Bateman A., Binns D., Bork P., Das U., Daugherty L., Duguenne L. et al. 2009: InterPro: the integrative protein signature database. Nucl. Acids Res. 37: D211-D215.

Kornbluth S. \& White K. 2005: Apoptosis in Drosophila: neither fish nor fowl (nor man, nor worm). - J. Cell Sci. 118 1779-1787.

LAVINE M.D. \& StRAND M.R. 2002: Insect hemocytes and their role in immunity. - Insect Biochem. Mol. Biol. 32: 1295-1309.

LIAO W.T., YANG Y. \& WU X.F. 2002: Expression and functional analysis of an inhibitor of apoptosis protein from Trichoplusia ni. - Biocheml. Biophys. Res. Commun. 293: 675-679.

MARTIN S.J. 2002: Destabilizing influences in apoptosis: sowing the seeds of IAP destruction. - Cell 109: 793-796.

Meier P., Silke J., Leevers S.J. \& Evan G.I. 2000: The Drosophila caspase DRONC is regulated by DIAP1. - EMBO J. 19: 598-611.

Meunier E., Dick M.S., Dreier R.F., Schurmann N., Kenzelmann B.D., Warming S., Roose-Girma M., Bumann D., KaYagaKi N., TAKEDA K. ET AL. 2014: Caspase-11 activation requires lysis of pathogen-containing vacuoles by IFN-induced GTPases. Nature 509: 366-370.

Opferman J.T. \& Korsmeyer S.J. 2003: Apoptosis in the development and maintenance of the immune system. - Nat. Immunol. 4: 410-415.

RiedL S.J. \& SHI Y.G. 2004: Molecular mechanisms of caspase regulation during apoptosis. - Nat. Rev. Mol. Cell Biol. 5: 897-907.

SALVeSEN G.S. \& DuCKETT C.S. 2002: IAP proteins: Blocking the road to death's door. - Nat. Rev. Mol. Cell Biol. 3: 401-410.

SeshagiRi S., Vucic D., Lee J. \& Dixit V.M. 1999: Baculovirusbased genetic screen for antiapoptotic genes identifies a novel IAP. - J. Biol. Chem. 274: 36769-36773.

SuzuKi M. \& TANAKA T. 2007: Development of Meteorus pulchricornis and regulation of its noctuid host, Pseudaletia separata. — J. Insect Physiol. 53: 1072-1078.

Tenev T., Zachariou A., Wilson R., Dizel M. \& Meier P. 2005: IAPs are functionally non-equivalent and regulate effector caspases through distinct mechanisms. - Nat. Cell Biol. 7: 70-77.

Thompson J.D., Higgins D.G. \& Gibson T.J. 1994: Clustal W: improving the sensitivity of progressive multiple sequence alignment through sequence weighting, position-specific gap penalties and weight matrix choice. - Nucl. Acids Res. 22: 4673-4680.

Vaux D.L. \& Korsmeyer S.J. 1999: Cell death in development. - Cell 96: 245-254.

VAuX D.L. \& SiLKe J. 2005:. IAPs, RINGs and ubiquitylation. Nat. Rev. Mol. Cell Biol. 6: 287-297.

Vilaplana L., Pascual N., Perera N. \& Belles X. 2007: Molecular characterization of an inhibitor of apoptosis in the Egyptian armyworm, Spodoptera littoralis, and midgut cell death during metamorphosis. - Insect Biochem. Mol. Biol. 37: 1241-1248.

Wang S.L., Hawkins C.J., Yoo S.J., Muller H.A.J. \& Hay B.A. 1999: The Drosophila caspase inhibitor DIAP1 is essential for cell survival and is negatively regulated by HID. - Cell 98: 453-463.

Wyllie A.H., Kerr J.F. \& Currie A.R. 1980: Cell death: the significance of apoptosis. - Int. Rev. Cytol. 68: 251-306.

Yokoi K., Kamezaki M., Yoshida T., Tanaka T. \& Miura K. 2013: Sensitivity to RNA interference-mediated gene silencing in intact and ligated larvae of the armyworm, Mythimna separata (Lepidoptera: Noctuidae). - Appl. Entomol. Zool. 48: 431-439.

Zhang J.Y., Pan M.H., Sun Z.Y., Huang S.J., Yu Z.S., Liu D., Zнао D.H. \& Lu C. 2010: The genomic underpinnings of apoptosis in the silkworm, Bombyx mori. - BMC Genomics 11: $611,17 \mathrm{pp}$.

Received February 27, 2018; revised and accepted April 20, 2018 Published online May 15, 2018 